\title{
NOTE
}

\section{The Twisted Cubic and Camera Calibration}

\author{
ThOMAS BUCHANAN \\ Advanced Development Communications Systems, Robert Bosch GmbH, Postfach 110261 , \\ D-6100 Darmstadt, Federal Republic of Germany
}

Received August 25, 1986; accepted October 30, 1987

\begin{abstract}
We state a uniqueness theorem for camera calibration in terms of the twisted cubic. The theorem assumes the general linear model and is essentially a reformulation of Seydewitz's star generation theorem. 1988 Academic Press, Inc.
\end{abstract}

This note points out a tie-in between the geometry of the twisted cubic and camera calibration. A similar observation has been made by [2, pp. 25-26] without mentioning special cases.

The relevant geometry was established by 19 th century geometers. The theorem stated below is essentially a reformulation of the star generation theorem of [6] (cf. [3, 2. Abtheilung; 7, Band II, pp. 172-183; 4, p. 309]).

By camera calibration we mean the determination of the parameters of the camera transformation using the coordinates of a set of "reference points" in 3-space and their corresponding image coordinates. In photogrammetry this is referred to as the "determination of the orientation."

A system of linear equations assuming the general linear model can be found for example in $[1$, p. 482]. Using this model we have the following:

THEOREM. Let $\mathscr{R}$ be a set of reference points in real 3-space. Let $C$ be a projective transformation of maximal rank from 3-space onto 2-space with $K=\operatorname{Ker} C$. Then there exists a projective transformation $C^{\prime}$, distinct from $C$, with $C(R)=C^{\prime}(R)$ for all $R \in \mathscr{R}$ if and only if

(a) $\mathscr{R} \cup\{K\}$ is a subset of a possibly composite twisted cubic or line.

(b) $\mathscr{R}$ is a subset of the set theoretic union of a line and a plane, and $K$ lies on the

For a readable proof of both implications in the real case including a discussion of the degenerate cases see [7, Band II, p. 172ff.] Compare also the analytical approach in $[5 ; 4$, p. 309]; the latter reference restricts itself primarily to complex space.

The curves which occur in (a) of the theorem are explicitly

(i) the proper twisted cubic, which is defined in terms of homogeneous coordinates via the parameter representation

$$
x_{0}: x_{1}: x_{2}: x_{3}=f_{0}(\theta): f_{1}(\theta): f_{2}(\theta): f_{3}(\theta)
$$




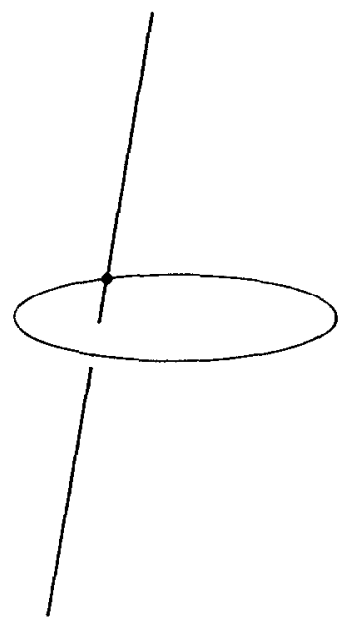

FIG. 1. A composite twisted cubic-a conic and a unisecant.

with

$$
f_{i}(\theta)=\sum_{j=0}^{3} a_{i j} \theta^{3-j}
$$

and $\operatorname{det}\left(a_{i j}\right) \neq 0$;

(ii) the union of a conic and a unisecant as indicated in Fig. 1;

(iii) the union of two skew lines and a transversal as indicated in Fig. 2.

In (ii) and (iii) the terms "unisecant" and "transversal" are used in their projective sense, i.e., the point of intersection may lie on the plane at infinity.

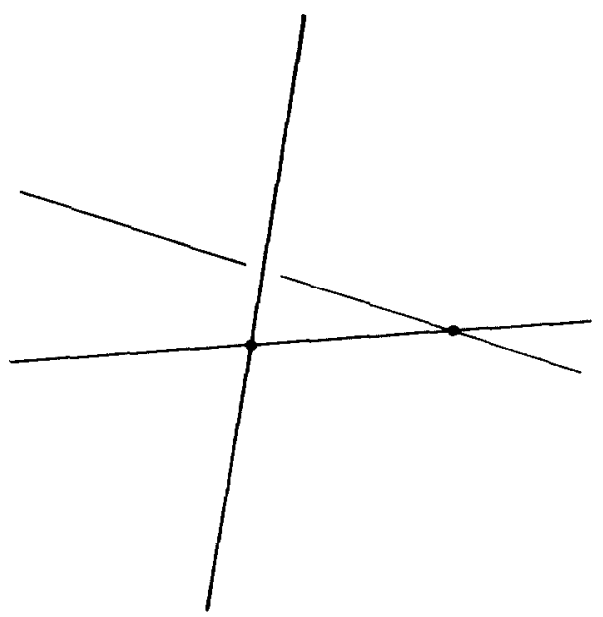

FIg. 2. A composite twisted cubic-two skew lines and a transversal. 
In applications $K=\operatorname{Ker} C$ corresponds to the position of the camera. The general assumptions of the theorem should be understood as stipulating that all reference points lie in the camera's field of view. The theorem implies that the coefficient matrix in the matrix equation for camera calibration given in $[1$, p. 482$]$ is not of maximal rank if and only if the lens and the reference points lie on the configurations as stated in the theorem. If the data is noisy, the theorem implies that this coefficient matrix is ill-conditioned when the reference points lie on one of the given configurations; conversely, it suggests that the matrix might be well-conditioned when these configurations are avoided.

Remark. Six points are insufficient to ensure unique camera calibration from all camera positions. This is a consequence of the fact that a possibly composite twisted cubic passes through any 6 points in space. See for example $[4, p$. 314] for an analytic proof of this when the points lie in general position. When 4 points lie in a plane, choose a possibly degenerate conic to pass through the 4 points and the point of intersection of the plane with the join of the remaining 2 points, and thus obtain a conic and a unisecant.

EXAMPLE. Take 7 points in 3-space, 4 in one plane, 3 in another such that no 3 lie on a line. If these are taken to be reference points, camera calibration is possible from any position from which all reference points are in the camera's field of view.

\section{ACKNOWLEDGMENT}

The author has profited from various suggestions from Professor Hermann Hähl from the University of Kiel, who was kind enough to comment on an early draft of this paper.

\section{REFERENCES}

1. D. H. Ballard and C. M. Brown, Computer Vision, Prentice-Hall, Englewood Cliffs, NJ, 1982.

2. S. Finsterwalder, Die geometrischen Grundlagen der Photogrammetrie, Jahresber. Deutsch. Math.Verein. 6 (2), 1897, 1-41.

3. T. Reye, Die Geometrie der Lage, 1. Aufl. Rümpler, Hannover, 1868; 2. Aufl. Baumgärtner, Leipzig, 1882; 3. Aufl. Baumgärtner, Leipzig, 1892; 4. Aufl. Kröner, Stuttgart, 1907.

4. J. G. Semple and G. T. Kneebone, Algebraic Projective Geometry, Oxford Univ. Press (Clarendon), Oxford, 1952.

5. F. Schur, Ueber die Horoptercurve, Dorpater Naturforscher-Gesellschaft; Sitzungsber. NaturforscherGesellschaft Univ. Dorpat 9, 1889, 162-164.

6. F. Seydewitz, Lineäre Konstruction einer Curve doppelter Krümmung, Arch. Math. Phys. 10 1847, 203-214.

7. R. Sturm, Die Lehre von den geometrischen Verwandtschaften, Teubner, Leipzig, Berlin, 1908. 